make them attractive also for the treatment of cover plates, bringing yet further benefits to the overall performance.

\section{Solar-powered Engines}

If the collector is employed as the heat source for a thermodynamic engine, say of the type shown in Figure 4, the effect of the surface treatment on the performance is equally marked (4). This is because the efficiency of a thermodynamic device is a function of the difference between the maximum and minimum temperatures of the cycle. As seen from Figure 3, the energy available from the collector falls as the output temperature rises, but the efficiency of the engine increases, so that the overall efficiency passes through a maximum at some temperature. This is illustrated in Figure 5, which shows clearly the strong effect of collector characteristics on the system efficiency (4).

The efficiency of a low-temperature system such as that of Figure 4 may be rather low to be of widespread value. It can be raised only by operating at higher temperatures and to obtain these the solar energy falling on a large area must be concentrated on to a smaller one. Two such schemes are illustrated in Figure 6. It will be seen that both involve reflection of solar radiation, as well as absorption with a selective coating as for the flat collector. In the more elaborate scheme (5) one reflective coating has to be applied on glass. Though gold films do not have a very high reflectivity at solar wavelengths, this can be obtained with some of its alloys $(1,2)$. It is possible that the great chemical stability of gold and gold alloys may give them a competitive advantage over other materials for long-term use in situations like these.

The fact that the desired selective optical properties may be obtained with films of such small thickness means that it becomes possible to consider using an expensive metal at quite modest cost. A place for gold films in solar energy utilisation seems assured.

\section{Acknowledgement}

Some of the illustrations for this paper are taken from the author's book (4) by agreement with the publisher, Compton-Russell Ltd, Salisbury, England.

\section{References}

1 R. C. Langley, Gold Bulletin, 1971, 4, 62-66

2 O. Loebich, Gold Bulletin, 1972, 5, 2-10

3 L. Harris, R. T. McGinnies and B. M. Siegel, F. Opt. Soc. Am., 1948, 38, 582-589

4 B. J. Brinkworth, Solar Energy for Man, Compton Press, 1972, ISBN 900193131

5 A. B. Meinel and M. P. Meinel, Physics Today, 1972, 25, $44-50$

\title{
The Tribological Behaviour of Gold
}

\section{A BASIC STUDY OF FRICTION AND WEAR}

The importance attached to the wearing and frictional properties of gold has increased greatly with its extensive applications in the form of sliding contacts in the electronics industry, and a number of papers have been published describing practical investigations in this field. A more fundamental study of the characteristics of a gold-to-gold system under carefully controlled rubbing conditions has recently been reported by Harmut Frey and Heinz-Gerhard Feller working in the Institut für Metallforschung in the Technical University of Berlin (Z. Metallkunde, 1973, 64, (8), 529-536).

The method employed was to cause a small cylinder of polycrystalline gold to be moved across the surface of a single crystal of gold, both being of 99.999 per cent purity. The depth and width of the wear track could be measured; so too could the size and nature of wear products.

It was observed that after an initial "wear-free" period of operation, characterised by a relatively low coefficient of friction which in turn had been shown to vary according to the crystallographic plane, the coefficient of friction between the two surfaces increased rapidly and a wear track appeared on the roller. Simultaneously gold was transferred from the roller to the single crystal and wear particles appeared. As the experiment continued the coefficient of friction decreased, as too did wear, until a condition more or less of equilibrium was established again with a relatively low coefficient of friction and insignificant wear. The "wear-free" period is of special significance. Its duration depends on load and so too does the nature of the wear track, and this must point to the advantages of keeping contact force to a minimum.

Examination of the wear products by scanning electron microscope showed that the form and size of the wear particles depended on test conditions. At light loads most of the metal transferred from the roller was in the form of small flat particles that were directly sheared off the parent surface. In time these broke down into flakes. Particle size also depended on load. In one example, with a load of $10 \mathrm{~g}$ the number of particles was more than four times as great as with $100 \mathrm{~g}$ although in the former case the mean diameter of the particles was barely one third.

The underlying causes of these phenomena are complex. The authors suggest that adhesion is responsible not only for friction but also for material transfer, although loose particles develop as a result of mechanical fatigue of the surfaces. The melting of individual microcontacts also plays a part in the overall phenomena. With this in mind they suggest that in further investigations of this nature attention should be directed to the basic principles of elastic and plastic deformation and fracture.

J. G. W. 